\title{
Behavioral and Structural Effects of Single and Repeat Closed-Head Injury
}

\author{
(D)Y.-C.J. Kao, (D)Y.W. Lui, (DC.-F. Lu, DH.-L. Chen, DB.-Y. Hsieh, and (D) C.-Y. Chen
}

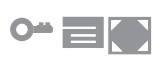

\begin{abstract}
BACKGROUND AND PURPOSE: The effects of multiple head impacts, even without detectable primary injury, on subsequent behavioral impairment and structural abnormality is yet well explored. Our aim was to uncover the dynamic changes and long-term effects of single and repetitive head injury without focal contusion on tissue microstructure and macrostructure.
\end{abstract}

MATERIALS AND METHODS: We introduced a repetitive closed-head injury rodent model $(n=70)$ without parenchymal lesions. We performed a longitudinal MR imaging study during a 50-day study period ( $T_{2}$-weighted imaging, susceptibility-weighted imaging, and diffusion tensor imaging) as well as sequential behavioral assessment. Immunohistochemical staining for astrogliosis was examined in a subgroup of animals. Paired and independent $t$ tests were used to evaluate the outcome change after injury and the cumulative effects of impact load, respectively.

RESULTS: There was no gross morphologic evidence for head injury such as skull fracture, contusion, or hemorrhage on micro-CT and MR imaging. A significant decrease of white matter fractional anisotropy from day 21 on and an increase of gray matter fractional anisotropy from day 35 on were observed. Smaller mean cortical volume in the double-injury group was shown at day 50 compared with sham and single injury $(P<.05)$. Behavioral deficits $(P<.05)$ in neurologic outcome, balance, and locomotor activity were also aggravated after double injury. Histologic analysis showed astrogliosis 24 hours after injury, which persisted throughout the study period.

CONCLUSIONS: There are measurable and dynamic changes in microstructure, cortical volume, behavior, and histopathology after both single and double injury, with more severe effects seen after double injury. This work bridges cross-sectional evidence from human subject and pathologic studies using animal models with a multi-time point, longitudinal research paradigm.

ABBREVIATIONS: $A D=$ axial diffusivity; $C D 68$ = Cluster of Differentiation $68 ; \mathrm{CHI}=$ closed-head injury; $\mathrm{FA}$ = fractional anisotropy; GFAP = glial fibrillary acidic protein; $\mathrm{MD}=$ mean diffusivity; $\mathrm{mNSS}=$ modified Neurological Severity Score; $\mathrm{RD}$ = radial diffusivity; $\mathrm{TBI}$ = traumatic brain injury

T raumatic brain injury (TBI) is a major public health issue with increasing incidence and enormous cost to society worldwide. ${ }^{1,2}$ Mild TBI is generally imperceptible on conventional diagnostic imaging, yet it can be associated with widespread symptoms. In patients with mild TBI, quantitative MR imaging analysis

Received December 17, 2018; accepted after revision February 16, 2019.

From the Neuroscience Research Center (Y.-C.J.K., C.-Y.C.) and Translational Imaging Research Center (Y.-C.J.K., C.-Y.C.), Taipei Medical University, Taipei, Taiwan; Department of Radiology (Y.-C.J.K., C.-Y.C.), School of Medicine, College of Medicine, Taipei Medical University, Taipei, Taiwan; Department of Radiology (Y.W.L.), NYU School of Medicine/NYU Langone Health, New York, New York; Department of Biomedical Imaging and Radiological Sciences (C.-F.L.), National Yang-Ming University, Taipei, Taiwan; Departments of Medical Research (H.-L.C.), Medical Imaging (C.-Y.C.), and Radiogenomic Research Center (Y.-C.J.K., C.-Y.C.), Taipei Medical University Hospital, Taipei, Taiwan; and Department of Biomedical Imaging and Radiological Science (B.-Y.H.), China Medical University, Taichung, Taiwan. This work was supported by the Ministry of Science and Technology, Taiwan (MOST105-2628-B-038-002-MY2, MOST104-2923-B-038-003-MY3, MOST106-2314B-010-058-MY2, MOST105-2221-E-038-007-MY3 and MOST107-2314-B-038-005), Taipei Medical University (TMU103-AE1-B27), and Taipei Medical University Hospital (105TMUH-SP-01, GH107SP02). assessing macrostructural and microstructural effects of injury has been extremely promising. ${ }^{3,4}$ Specifically, human subject studies suggest regional cortical volume $\operatorname{loss}^{5,6}$ and abnormal fractional anisotropy (FA) in white matter regions susceptible to traumatic axonal injury, such as the corpus callosum, internal and external capsules, and anterior and posterior corona radiata. ${ }^{4,7}$ More recently, multishell diffusion with biologic modeling suggests WM pathologic changes. ${ }^{8}$ However, due to the difficulties of performing multiple, rapid, serial imaging studies as well as the

This work originated from Taipei Medical University (Address: 250 Wuxing St., Taipei 110, Taiwan).

Please address correspondence to Cheng-Yu Chen, MD, Department of Radiology, School of Medicine, College of Medicine, Taipei Medical University, 250 Wuxing St., Taipei 110, Taiwan; e-mail: sandy0932@gmail.com; @sandy0932

- Indicates open access to non-subscribers at www.ajnr.org

三 Indicates article with supplemental on-line appendix and tables.

Indicates article with supplemental on-line photos.

http://dx.doi.org/10.3174/ajnr.A6014 
lack of histopathologic confirmation in human subjects with concussion, we still lack a clear understanding of the evolution of tissue injury across time. Furthermore, there is a specific, growing need to elucidate the effect of multiple head impacts/head injuries on short-term recovery and long-term outcome.

The purpose of this work was to measure dynamic temporal changes of macrostructural and microstructural damage from the acute-to-chronic phase postinjury after both single and repetitive head injury using a well-controlled model of closed-head injury (CHI) without focal contusion/hemorrhage. We describe observed differences among animals after single injury, double injury, and sham surgery and report the behavioral and neuropathologic correlates of injury.

\section{MATERIALS AND METHODS}

The study was performed in accordance with the recommendations of National Institutes of Health Guidelines for Animal Research (Guide for the Care and Use of Laboratory Animals) and the Animal Research: Reporting In Vivo Experiments guidelines. The protocol was approved by the Institutional Animal Care and Use Committee at Taipei Medical University. Animals were randomly assigned to 3 groups (single, double CHI, and sham control) for longitudinal and cross-sectional assessment (On-line Fig $1)$.

\section{Closed-Head Injury Model}

Seventy adult male Sprague-Dawley rats weighing 250-400 g (BioLASCO Taiwan Co, Taipei City, Taiwan) were anesthetized using chloral hydrate $(400 \mathrm{mg} / \mathrm{kg})$ and placed on a stereotaxic frame for an operation. All surgical procedures were performed under aseptic conditions. This $\mathrm{CHI}$ model modifies the protocol from the weight-drop injury model ${ }^{9}$ using the following: 1) fixed head positioning to achieve precise localization and force delivery, 2) addition of a secured impactor, and 3) alteration of the height of a freely dropped brass weight. Briefly, a midline incision was performed on the scalp, and a circular stainless steel helmet (10-mm diameter) was cemented over the skull on top of the left sensorimotor cortex ( $1.5 \mathrm{~mm}$ posterior and $2.5 \mathrm{~mm}$ lateral to the bregma). A 600-g weight was dropped from a height of $1 \mathrm{~m}$ through a stainless steel tube (1-m height with an inner diameter of $20 \mathrm{~mm}$ for clearing a column of stainless brass weights) to the secured impactor with a round tip aimed at the metal helmet. Animals in the double CHI group received 2 impacts with a 1-hour interval. ${ }^{10}$ The helmet was immediately removed after the last impact, and the scalp was sutured. Physiologic parameters, including heart rate and blood oxygen saturation level, were continuously monitored using a pulse oximeter (SurgiVet; Smiths Medical, Waukesha, Wisconsin). Rats were given topical antiseptics and lidocaine at the surgical site during the postoperative period.

\section{CT}

Micro-CT images were obtained on harvested skulls using a SkyScan 1176 scanner (Bruker BioSpin, Ettlingen, Germany) at an isotropic resolution of $9 \mu \mathrm{m}$ and were visually assessed for subtle skull fractures by a neuroimaging expert.

\section{MR Imaging}

Longitudinal MR imaging was performed in each group before, at 24 hours, 7 days, 21 days, 35 days, and 50 days after CHI or sham surgery using a PharmaScan 7T system (Bruker BioSpin). Animals were anesthetized under $\sim 1.2 \%$ isoflurane, and the stereotaxic headpiece and holder, consisting of ear and tooth bars, were used to immobilize the head. Physiologic conditions, including heart rate, arterial pulse extension, oxygen saturation, and rectal temperature, were continually monitored and maintained within normal ranges throughout the experiment. ${ }^{11-13}$ A volume coil was used for radiofrequency excitation, and an array coil was used for signal receiving. Initial localization scans were obtained, and $\mathrm{T}_{2}$ weighted images using a rapid acquisition with a relaxation enhancement sequence $(\mathrm{TR} / \mathrm{TE}=3600 / 40 \mathrm{~ms}, \mathrm{FOV}=2.0 \times 2.0 \mathrm{~cm}$, matrix size $=256 \times 256,16$ slices, slice thickness $=1 \mathrm{~mm}$ ) were obtained to acquire anatomic images for rodent models. ${ }^{11,13}$ Diffusion tensor images were acquired with the same geometry using the 4 -shot spin-echo EPI with $\mathrm{TR} / \mathrm{TE}=3000 / 28 \mathrm{~ms}$, matrix size $=$ $96 \times 96, \delta / \Delta=5 / 15 \mathrm{~ms}$, number of $\mathrm{B}_{0}=5$, number of directions $=30, \mathrm{~b}$-value $=1000 \mathrm{~s} / \mathrm{mm}^{2}$, number of averages $=4$. Susceptibility-weighted images were obtained with the same geometry using the flow-compensated gradient-echo sequence with bandwidth $=30 \mathrm{kHz}, \mathrm{TR} / \mathrm{TE}=600 / 18 \mathrm{~ms}$, matrix size $=256 \times$ 256, flip angle $=40^{\circ}$, and number of averages $=6$.

\section{Image Analysis}

$\mathrm{T}_{2}$-weighted imaging and SWI were visually inspected for brain deformation, tissue loss, edema, and the presence of hemorrhage by 1 attending neuroradiologist with $>25$ years of experience and 1 neuroimaging expert with 5 years of experience in rodent models. Image analysis, including skull stripping and motion correction/coregistration across time points and subjects, was performed using Statistical Parametric Mapping software (http:// www.fil.ion.ucl.ac.uk/spm/software/spm12) and a custom Matlab (MathWorks, Natick, Massachusetts) script, published previously. ${ }^{11,12,14}$ To measure the cortical volume, we manually drew cortical ROIs and then applied them onto coregistered $\mathrm{T}_{2}$ weighted images. To better delineate the border between the cortex and WM, we applied a threshold of 2 SDs of the signal intensity of cortical ROIs. All threshold-defined results were visually inspected for gross errors by 2 experienced investigators blinded to the experimental groups.

Parametric diffusion maps were generated for mean diffusivity $(\mathrm{MD})$, axial diffusivity $(\mathrm{AD})$, radial diffusivity ( $\mathrm{RD})$, and FA. Rectangular ROIs $\left(5 \times 2.5 \mathrm{~mm}^{2}\right.$ per slice $)$ were manually placed on FA maps on 3 consecutive slices subjacent to the impact region in the cortex, WM, and subcortical gray matter (Fig 1A). A threshold of FA $>0.35$ was applied to extract WM. ${ }^{14}$ Cortical and subcortical tissue was segmented using a pixel-by-pixel classification based on spatial information relative to WM. Rectangular ROIs $\left(1 \times 1.5 \mathrm{~mm}^{2}\right.$ per slice $)$ were manually placed on FA maps on 3 consecutive slices (3-5 mm posterior to the bregma) covering the ipsilateral hippocampus. Mean $\mathrm{MD}, \mathrm{AD}, \mathrm{RD}$, and FA values were calculated in the hippocampal ROIs and WM and cortical and subcortical tissue in the ROIs adjacent to the impact region. 
A
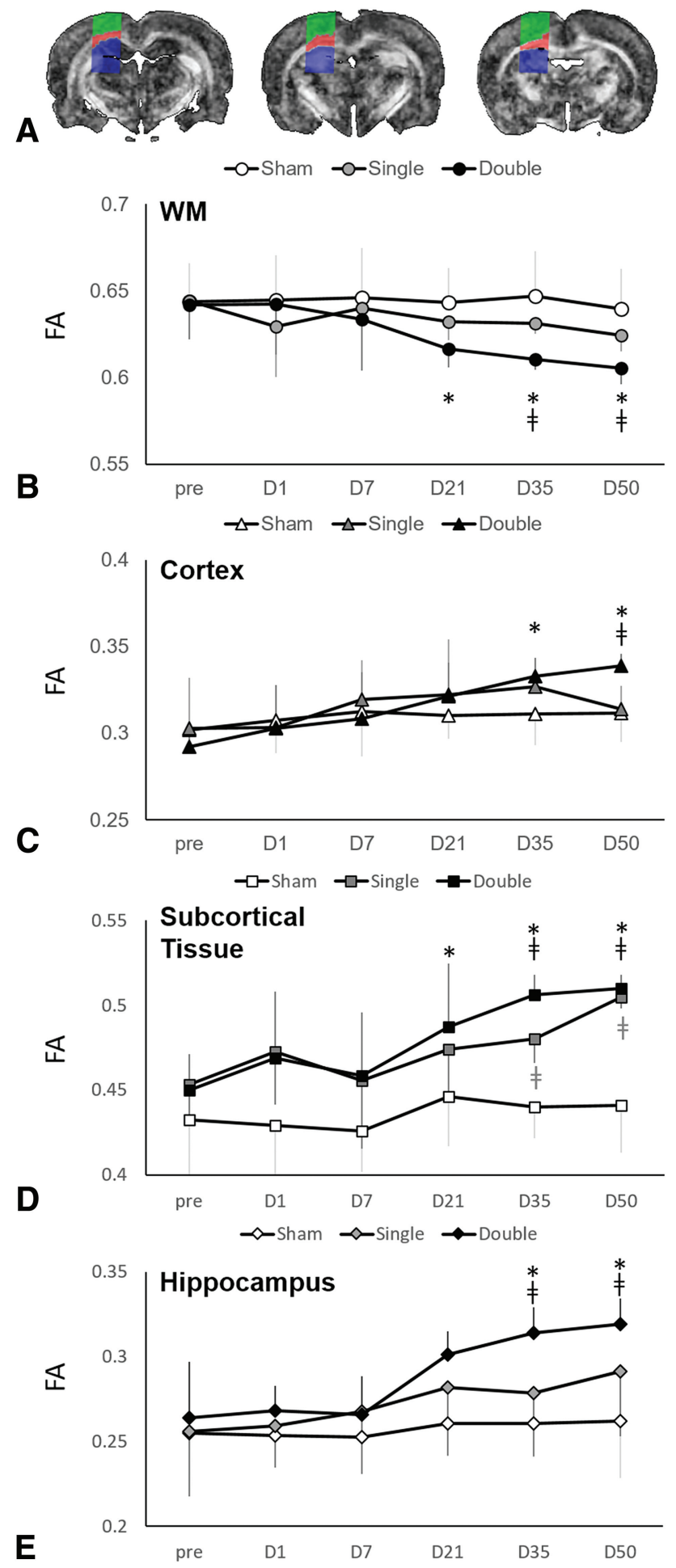

FIG 1. Longitudinal changes in FA after $\mathrm{CHI}$. A, Automatically segmented ROls deep to the site of impact on 3 consecutive slices show the cortex (green), white matter (red), and subcortical tissue (blue). Longitudinal follow-up during the first 50 days shows the evolution of mean FA values across time, which differ between white matter $(B)$ and gray matter $(C-E)$ structures, as well as important differences between single and double injury. Data are means; $₫$ indicates $P<.05$ versus the sham; asterisk, $P<.05$ versus baseline (day 0 [D0]).

\section{Behavioral Assessment}

Assessments of the modified Neurological Severity Score (mNSS), the Beam Walk Balance Test, and open-field test were performed before, at 24 hours, and every 7 days after $\mathrm{CHI}$ or sham surgery. The mNSS evaluates motor sensory reflex, muscular status, and balance. ${ }^{10,15}$ Points are awarded for the inability to perform the task, the lack of reflex, or abnormal response with a maximum possible score of 20 (higher score indicates worse outcome.) The Beam Walk Balance Test measures the time required for the animal to cross the beam, assessing balance and coordinated motor function. ${ }^{16}$ Animals who fell off the beam or did not finish the task received a score of 180 seconds The open-field test was used to assess spontaneous activity and anxiety-like behavior. ${ }^{17}$ Tracking and calculation of the movement duration, mean travel distance, center entries, and center time of each animal during the trial were analyzed using ActualTract software (Actual Analytics, Edinburgh, United Kingdom) (On-line Appendix). ${ }^{18}$

\section{Histology}

Immunohistochemical staining of formalin-fixed, paraffin-embedded tissue sections was performed using the Immunoperoxidase Secondary Detection system kit (IHC Select, DAB500; Millipore, Temecula, California). Briefly, the slides were deparaffinized and treated with $3 \% \mathrm{H}_{2} \mathrm{O}_{2}$ to block endogenous peroxidase activity. Citrate buffer was used for antigen retrieval at $90^{\circ} \mathrm{C}$. Staining procedures were performed according to manufacturer's recommendation. Specimens were counterstained with hematoxylin and mounted with ProLong Gold Antifade reagent (P36930; ThermoFisher Scientific, Waltham, Massachusetts). To assess neuroinflammation, we used the following antibodies for immunohistochemical staining: anti-glial fibrillary acidic protein (GFAP) (ab68428; Abcam, Cambridge, Massachusetts) and antiCluster of Differentiation 68 (CD 68) (GTX41868; GeneTex, Irvine, California).

All images were acquired using a DP 80 light microscope slide scanner (Olympus, Shinjuku, Japan). ROIs of the ipsilateral cortex ( $2 \mathrm{~mm}$ posterior to the bregma) and the CA1 region of the ipsilateral hippocampus ( $3 \mathrm{~mm}$ posterior to the bregma) were traced in 3 consecutive sections from each animal using cellSens Dimension software (Olympus America, Center Valley, Pennsylvania). ${ }^{10}$ The number of activated astrocytes and microglia in the ROIs was counted using FIJI/ImageJ software (National Institutes of Health, Bethesda, Maryland). ${ }^{19}$

\section{Statistical Analysis}

Statistical analyses were performed using SPSS software (IBM, Armonk, New York). Significant differences in DTI metrics, cortical volume, and behavioral outcome between sham, single, and repetitive injuries across the entire study period were assessed using a 2-way mixed ANOVA. Significant differences in GFAP and CD 68 expression were assessed using 2-way ANOVA. Post hoc tests were performed by the Tukey honestly significant difference and the Dunnett T3 post hoc tests for data passing and not passing the homogeneity test, respectively. The significance level was set at $P<.05$.

\section{RESULTS}

Inspection of $\mathrm{T}_{2}$-weighted imaging and SWI revealed no focal brain contusions or hemorrhage at both 24 hours and 50 days 
Single

T2WI
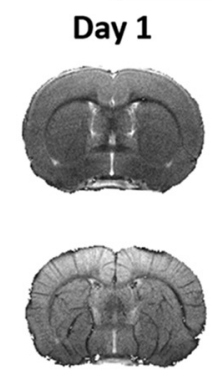

A

SWI

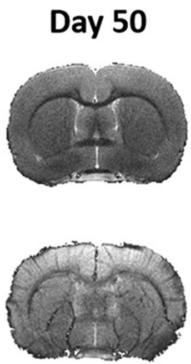

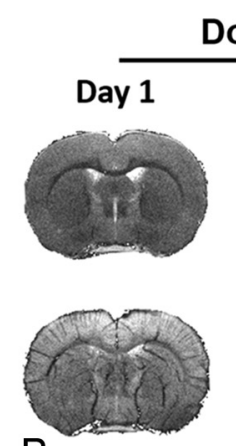

$\mathrm{B}$
Double

FIG 2. No focal hemorrhage or contusion on conventional MR imaging after experimental CHI. $\mathrm{T}_{2}$-weighted imaging and susceptibility-weighted imaging with in-plane resolution of $78 \mu \mathrm{m}$ obtained at 7T on day 1 and day 50 after single $(A)$ and double $(B) \mathrm{CHI}$ show no contusion, edema, or microhemorrhage. One representative animal from each group for the longitudinal study is shown here.
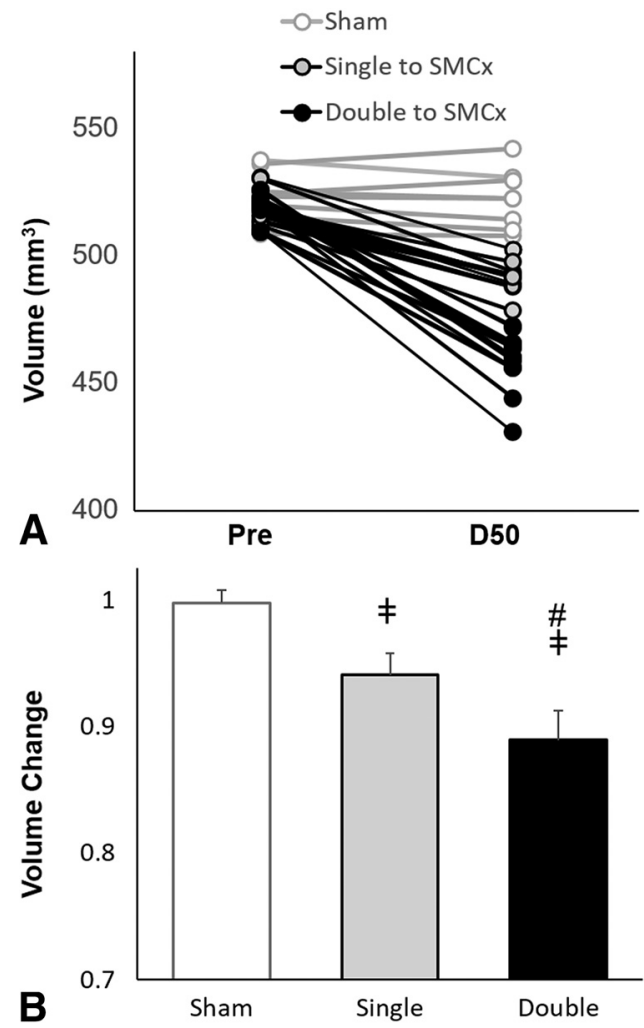

FIG 3. Cortical loss after experimental $\mathrm{CHI}$. There is a clear decrease in cortical volume as measured on $\mathrm{T}_{2}$-weighted images 50 days postinjury compared with baseline $(A)$, with a greater percentage volume change after double injury $(n=12)$ compared with sham surgery $(n=9)$ and single injury $(n=12)$. Reduction of cortical volume is associated with the number of impacts. Data are means. $t$ indicates $P<.05$ versus the sham; \#, $P<.05$ versus the single $\mathrm{CHI}$; SMCx, sensorimotor cortex.

postinjury (Fig 2), and there were no fractures identified using micro-CT.

\section{Cortical Volume Assessment}

There were no differences in mean baseline cortical volume among groups $(520.169 \pm 6.77,516.88 \pm 6.53$, and $523.68 \pm$ $10.48 \mathrm{~mm}^{3}$ for single injury, double injury, and sham groups, respectively). Longitudinal follow-up during 50 days revealed decreased cortical volume in the single- and double- injury groups $(P<.01)$. Furthermore, animals in the double-injury group sustained a significantly larger percentage change in volume $(0.11 \pm 0.02$ $\mathrm{mm}^{3}$ ) compared the single-injury group at 50 days follow-up $(0.06 \pm$ $\left.0.02 \mathrm{~mm}^{3}\right)(P<.01$; Fig 3$)$.

\section{Longitudinal Diffusion MR Imaging Changes}

Compared with baseline measurements, while there were no significant changes in MD after sham surgery or injury in all groups (On-line Table 1), significant changes in $\mathrm{AD}$ and $\mathrm{RD}$ after injury are shown in On-line Fig 2. There were differences in FA compared with baseline measures in the double-injury group but no change in FA with time in the sham and single-injury group (On-line Table 2). Namely, WM FA showed a significant decrease from the baseline value beginning at 21 days $(P=.04)$ after double injury, which was sustained at 50 days. In the cortex, there was a significant increase in FA from baseline beginning at 35 days $(P<.01)$ after double injury, sustained at 50 days. Subcortical FA showed a significant increase from baseline at 21 days $(P=.02)$ after double injury. Hippocampal FA showed a significant increase from baseline at 35 days $(P=.02$; Fig 1$)$ after double injury.

\section{Cross-Sectional Diffusion MR Imaging Between-Group Comparisons}

While there were no differences in MD among groups, significant changes in cortical AD and cortical RD were found between single- and double-injury groups after 35 days (On-line Fig 2). Higher mean subcortical FA $(P<.01)$ was observed in the singleinjury group compared with the sham group beginning 35 days after injury. Similarly, FA was higher in the double-injury group compared with the sham group in the cortical (at 50 days), subcortical, and hippocampal (beginning at 35 days) regions. FA was lower in the double-injury group compared with the sham group in WM (beginning at 35 days) $(P<.01$; Fig 1$)$. No other differences were seen among groups in terms of diffusion metrics.

\section{Behavioral Assessment}

All groups (sham and single- and double-injury) demonstrated significantly increased mNSS 24 hours after ictus (from $1.50 \pm$ 1.09 to $3.00 \pm 1.48$ for sham, from $2.67 \pm 0.58$ to $4.42 \pm 2.07$ for single injury, and from $3.00 \pm 1.41$ to $6.21 \pm 2.75$ for double injury; $P<.03$ ). mNSS in the sham and single-injury groups recovered after days 7 and 28, respectively; however, it continued to be elevated in the double-injury group for the entire study period $(P<.02)$. Animals in the double-injury group demonstrated higher mean mNSS compared with the single-injury $(P<$ $.01)$ and the sham $(P<.01)$ groups from 7 days on (Fig $4 A)$.

Beam-walking duration was significantly increased over baseline (28.50 \pm 2.90 seconds) beginning at 24 hours $(65.90 \pm 23.00$ seconds; $P<.01$ ) following double injury and remained increased during the entire 50 -day study period $(155.30 \pm 28.59$ seconds; 

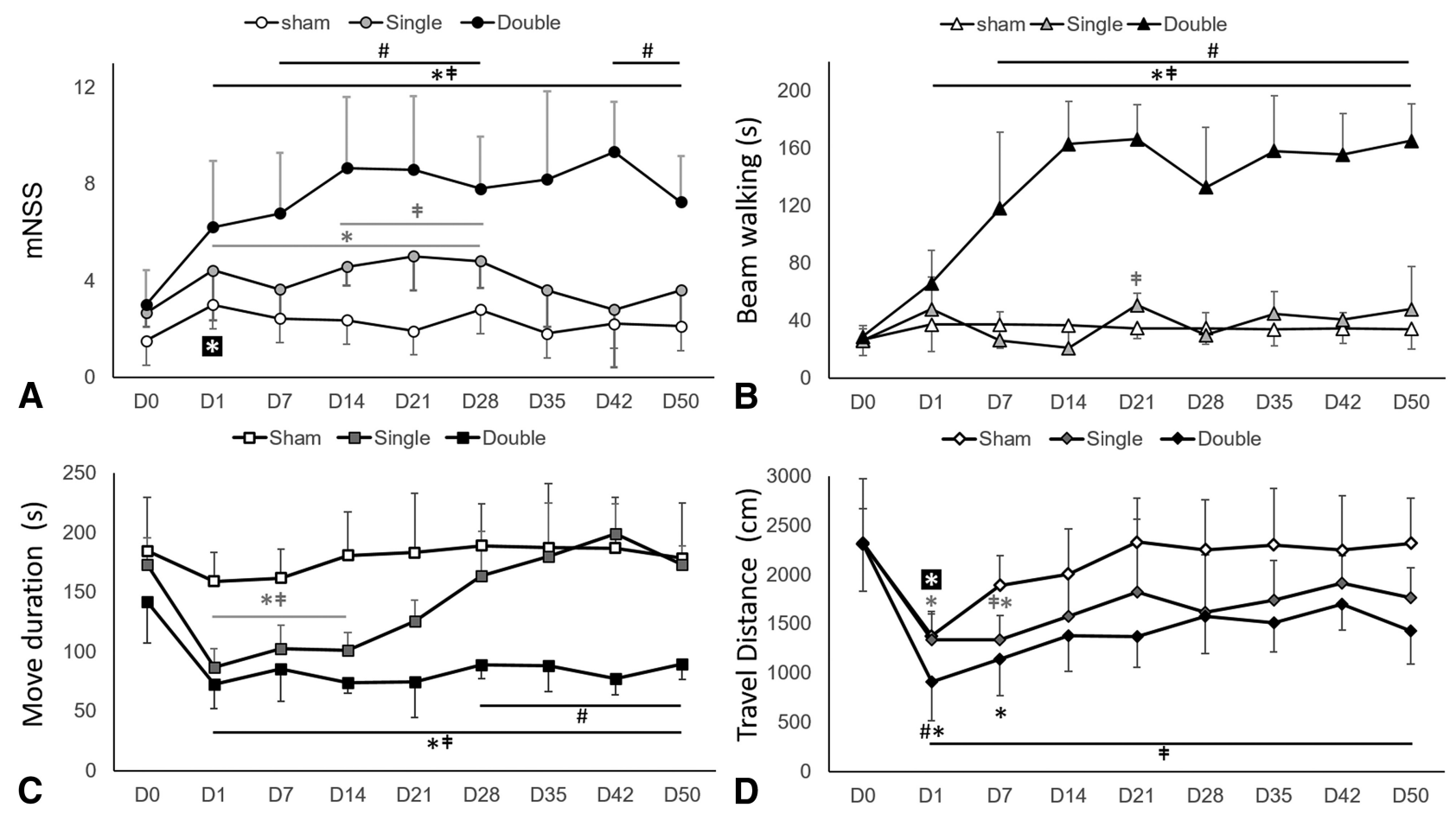

FIG 4. Behavioral deficits after CHI. mNSS $(A)$ and beam-walking duration $(B)$ after single and double $\mathrm{CHI}$ compared with sham surgery. In single-injury rodents, the mNSS score and beam-walking duration follow a similar time course compared with animals in the sham group; whereas in double-injury rodents, the mNSS score and beam-walking duration both increase significantly after $\mathrm{CHI}$ by day 7 and do not resolve by day 50 . Locomotive activity as measured by movement duration $(C)$ and travel distance $(D)$ in the open-field trial after single and double $\mathrm{CHI}$ is impaired and persisted at day 50 after double injury. Data are means. f indicates $P<.05$ versus the sham; asterisk, $P<.05$ versus baseline (day 0 [D0]); $\#, P<.05$ versus the single $\mathrm{CHI}$.

$P=.02)$. Significantly higher average beam-walking duration was observed after double injury (118.00 \pm 13.11 seconds) compared with single injury $(26.00 \pm 19.69$ seconds; $P<.01)$ after 7 days (Fig $4 A,-B$ ). There were no other significant differences in beamwalking duration scores.

With regard to the open-field test, both single- and doubleinjury groups showed decreased movement duration compared with baseline, beginning at 24 hours after CHI (from $173.30 \pm$ 22.00 to $86.67 \pm 15.93$ seconds for single injury; and from $141.92 \pm 34.63$ to $72.36 \pm 21.18$ seconds for double injury; $P<$ $.01)$. While movement duration in animals with single injury recovered after 14 days, movement duration in animals with double injury remained significantly lower compared with baseline for the entire 50 -day period $(77.27 \pm 12.50$ seconds; $P<.01)$. Between-group differences were seen in movement duration between sham (161.67 \pm 23.15 seconds) and single injury (102.34 \pm 19.90 seconds; $P<.01)$ as well as sham and double injury $(85.17 \pm 27.13$ seconds; $P<.01)$ from day 7 through 14 . This difference persisted in the double-injury group $(178.23 \pm 46.69$ versus $89.93 \pm 12.73$ seconds at day $50 ; P<.01)$, whereas the single-injury animals recovered movement duration scores in a manner more similar to sham animals beyond day 28. From day 28 on, there was also a significant difference between single $(163.83 \pm 37.31$ seconds $)$ and double $(88.59 \pm 11.45$ seconds; $P<$ .01 ) injury groups (Fig 4C).

Travel distance was also measured from the open-field test and was decreased in all groups from baseline measures at 24 hours (from $2314.14 \pm 657.09$ to $1378.06 \pm 224.62 \mathrm{~cm}$ for sham, from $2323.30 \pm 342.68$ to $1340.20 \pm 286.26 \mathrm{~cm}$ for single injury, and from $2308.50 \pm 479.38$ to $906.61 \pm 389.05 \mathrm{~cm}$ for double injury; $P<.03)$. In sham animals, the mean travel distance recovered by day 7 after surgery. In animals with single and double injury, travel distance recovered by day 14 after injury. While differences in travel distance between sham and single-injury groups were seen only on day 7 after CHI, statistical differences between the double-injury and sham groups were consistently present from 24 hours through day $50(P<.01)$. In addition, a difference in travel distance was also present between single- and double-injury groups initially at 24 hours $(P=.02)$ (Fig $4 D)$. To evaluate anxiety-like behavior, we measured center entries and center time from the open-field test (On-line Fig 3).

\section{Immunohistology}

Evidence for reactive gliosis was seen using GFAP and CD 68 immunohistologic staining in both the cortex and the CA1 region of the hippocampus after $\mathrm{CHI}$ (Fig $5 A$ ). GFAP immunoreactivity was observed in the cortex and hippocampus in both single- and double-injury animals compared with the sham animals at 24 hours and was sustained at 50 days $(P<$ $.05)$. Involvement of the hippocampus confirms that more diffuse histopathologic changes occurred remote from the direct site of impact. CD 68 expression was also observed in the cortex at 24 hours after double injury $(P=.01)$ and increased at day 50 after single and double injury compared with sham injury $(P<.02)$. CD 68 expression in the hippocampus was prominent compared with the sham animals at 50 days after double injury $(P<.02)$ (Fig $5 B-E)$. 

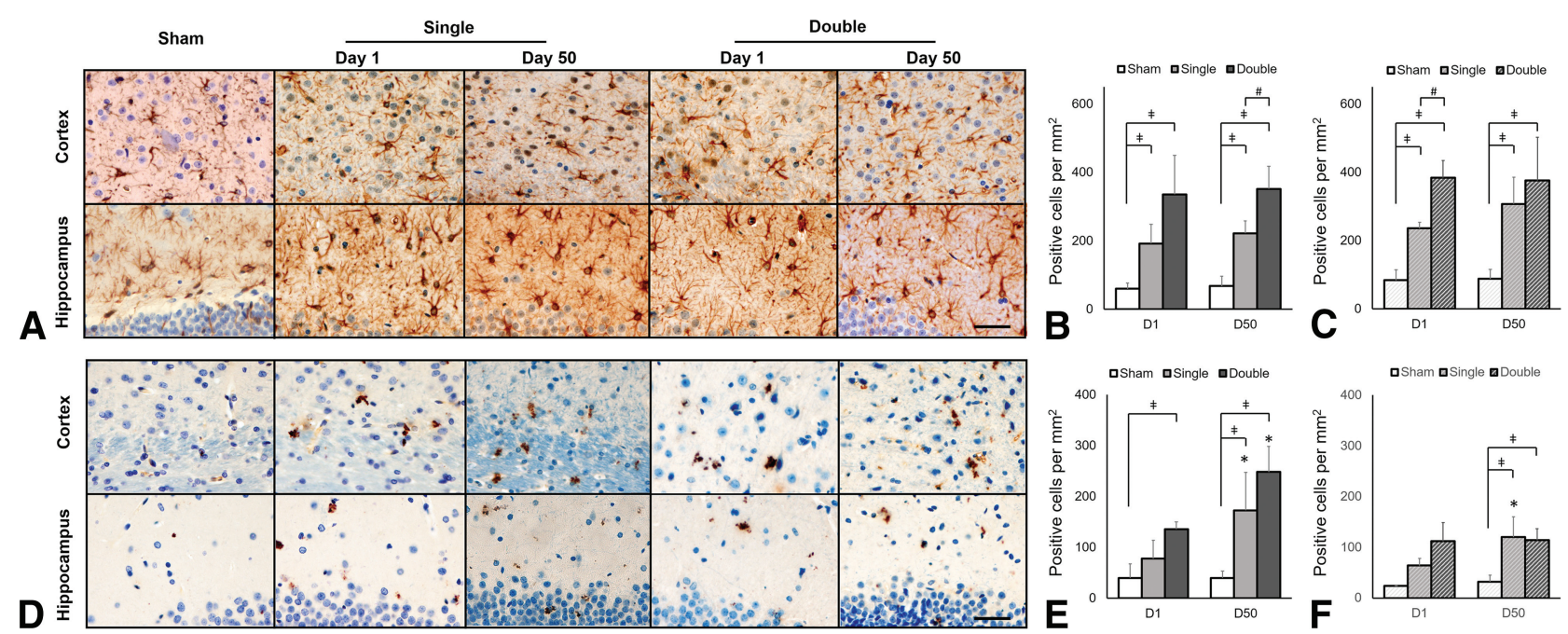

FIG 5. Astroglial and microglial expression in the cortex and hippocampus underlying the impact region after CHI. A, GFAP-positive staining and quantification of GFAP expression in the cortex (B) and hippocampus (C) after CHI. D, CD 68-positive staining and quantification of CD 68 expression in the cortex $(E)$ and hippocampus $(F)$ after CHI. As early as 24 hours after both single and double CHI, elevated GFAP is observed in both the cortex and hippocampus deep to the impact site. CD 68 expression indicating neuroinflammation is also observed at 24 hours postinjury and sustained to day 50 in the cortex after double $\mathrm{CHI}$. Scale bar, $40 \mu \mathrm{m}$.

\section{DISCUSSION}

Here we introduce a modified weight-drop model with fixed head positioning to allow a high-degree of control over the strength and location of impact, improving reproducibility of the injury. ${ }^{20,21}$ Our results show this to be a reliable model to deliver experimental CHI without focal traumatic brain lesions, a challenge and limitation of many prior studies of head injury using rodent models. ${ }^{20-22}$ This work provides the most comprehensive longitudinal study to date of single and double head injury using a reliable CHI model and seeks to fill in gaps in our knowledge regarding the temporal evolution of injury and recovery of the brain. We found significant macrostructural, microstructural MR imaging changes, behavioral changes, and histologic differences after injury in both single- and double-injury groups during the first 50 days after injury and describe these temporal changes.

Limited previous studies have reported brain atrophy in experimental TBI; most used a cross-sectional design with potential confounders such as ventricular dilation or tissue loss. ${ }^{23,24} \mathrm{Re}-$ cently, Meconi et $\mathrm{al}^{25}$ studied animals on day 7 after repeat awake $\mathrm{CHI}$ and reported no cortical volume loss. We observed a decrease in cortical volume during a longer follow-up period of 50 days, and it might be expected that macrostructural volume loss would be better detected at longer follow-up times as in other neurodegenerative animal models. Moreover, no significant change in the body weight of animals was observed at 50 days after injury compared with the baseline, suggesting that the cortical volume reduction was not associated with dehydration or malnutrition in our animals. Of note, cortical volume was reduced after both single and double injury, and the degree of atrophy was higher after double injury. This finding is in keeping with what we know from clinical experience and human subject research. Cortical atrophy is known to be a major neuropathologic feature of chronic TBI and has been shown in longitudinal studies to occur even after a single mild injury in humans. ${ }^{3}$ Multiple head injuries, even without focal acute hemorrhage or contusion, are associated with a long-term risk for a number of neurodegenerative disorders asso- ciated with brain volume loss, including chronic traumatic encephalopathy. ${ }^{26}$ We found increased cortical GFAP expression as early as 24 hours, which was sustained for 50 days, and we hypothesize that persistent neuroinflammation could predispose to neurodegeneration. ${ }^{27}$

We found significant changes in cortical volume and FA from baseline after both single and double injury, with differences between the 2 groups suggesting that injury to the brain may be cumulative. Specifically, our results show decreased WM FA after 21 days in the double-injury group. This is in keeping with most human studies of mild TBI that showed decreased FA in WM after injury. ${ }^{28}$ Previous animal studies of head injury (with contusion) also showed significantly reduced FA in the WM within 1 week of injury, which investigators have related to diffuse axonal injury and loss of myelin. ${ }^{29-32}$ We also found increased GM FA after double CHI in cortical, subcortical, and hippocampal structures. Published evidence of FA changes in gray matter after brain injury is mixed. ${ }^{17,31,33}$ The mechanism behind increased GM FA in TBI models is incompletely understood, though some have suggested this to be reflective of inflammatory changes ${ }^{29,30,32}$ and the orientation of astrocyte processes ${ }^{34,35}$ associated with underlying injury. We did, in fact, observe astrogliosis on immunohistochemical staining in this study. Future work combining atlas-based tissue segmentation and interrogation of a greater number of regions, including those more remote from the injury, will better illustrate the dynamic changes in diffusion anisotropy.

In terms of behavioral changes, worse outcome seen after double injury compared with single injury supports the idea that there is cumulative injury after multiple impacts sustained within a close time period. We found significant deficits in beam walking in animals who sustained double injuries, whereas the performance of animals in the single-injury group approximated the sham group closely at all time points. The clinical correlate may be slower walking speed and multijoint gait abnormalities reported in patients with chronic TBI. ${ }^{36}$ In addition, in contrast to the recovery of activity in the single-injury paradigm at 28 days, 
movement duration after double injury remained short, suggesting prolonged recovery after double injury. ${ }^{10}$

Our histopathologic findings confirm that astrogliosis is initiated within 1 day of injury, in keeping with previously published reports. ${ }^{10,19}$ Clinically, there is an association between elevated serum GFAP and the severity of head trauma in patients within 72 hours of injury. ${ }^{37}$ Taken together, our results support the presence of neuroinflammation occurring shortly after CHI, even with the absence of acute focal macrostructural injury, and this finding is in agreement with studies that point to serum GFAP as a promising noninvasive biomarker in the setting of even mild TBI. $^{38,39}$

Limitations of the current study include the use of a fixed head position during impact, which, while advantageous to quantify the delivery of impact, lacks the linear translation and rotation common to clinical scenarios of mild TBI. Other animal models are, however, criticized for the inability to quantify and control rebound effects as well as the common occurrence of focal hemorrhage/contusion. ${ }^{21,22}$ We have work currently underway to establish a related setup using unrestrained animals to better model acceleration-deceleration influences on injury. Our results demonstrate that CHI-related alterations are augmented by the number of impacts, though we studied only 1 repetitive paradigm. Additional studies using varying impact regions and repetition schemes would optimally take advantage of a model such as the one proposed here. Last, in addition to neuroinflammation, changes in the neural density, axonal integrity, or abnormal protein accumulation may also contribute to alteration in DTI metrics. ${ }^{31,40}$ We plan to examine this possibility using respective immunohistochemical stains in our future studies.

\section{CONCLUSIONS}

Our study describes a repetitive CHI model without acute macroscopic damage that leverages the benefits of controlling impact degree, location, and number. There are measurable neuroimaging abnormalities after $\mathrm{CHI}$ that evolve dynamically during the first 50 days postinjury, occurring after the immediate effects on behavior and neuropathology. There is a cumulative effect that the number of injuries has on later cortical atrophy, evolving microstructural changes, and neurobehavioral outcome in the first 50 days following injury. This work bridges predominantly crosssectional evidence from human subject studies and pathologic studies using animal models with a rich, multi-time point, longitudinal research paradigm.

Disclosures: Yu-Chieh Jill Kao-RELATED: Grant: Ministry of Science and Technology, Republic of China. Cheng-Yu Chen-RELATED: Grant: Ministry of Science and Technology, Republic of China. Comments: 3-year grant for animal model study.* Yvonne W. Lui-UNRELATED: Grants/Grants Pending: National Institutes of Health (R01 NS039135-11, R21 NS090349 and P41 EB017183)*; OTHER RELATIONSHIPS: Siemens-New York University collaboration lead and advisor for Bold Brain Ventures LP. *Money paid to the institution.

\section{REFERENCES}

1. Sosin DM, Sniezek JE, Thurman DJ. Incidence of mild and moderate brain injury in the United States, 1991. Brain Inj 1996;10:47-54 CrossRef Medline

2. Roozenbeek B, Maas AI, Menon DK. Changing patterns in the epi- demiology of traumatic brain injury. Nat Rev Neurol 2013;9:231-36 CrossRef Medline

3. Chong CD, Schwedt TJ. Research imaging of brain structure and function after concussion. Headache 2018;58:827-35 CrossRef Medline

4. Wu X, Kirov II, Gonen O, et al. MR imaging applications in mild traumatic brain injury: an imaging update. Radiology 2016;279:693707 CrossRef Medline

5. Cole JH, Leech R, Sharp DJ, et al; Alzheimer's Disease Neuroimaging Initiative. Prediction of brain age suggests accelerated atrophy after traumatic brain injury. Ann Neurol 2015;77:571-81 CrossRef Medline

6. Coughlin JM, Wang Y, Munro CA, et al. Neuroinflammation and brain atrophy in former NFL players: an in vivo multimodal imaging pilot study. Neurobiol Dis 2015;74:58-65 CrossRef Medline

7. Castaño Leon AM, Cicuendez M, Navarro B, et al. What can be learned from diffusion tensor imaging from a large traumatic brain injury cohort? White matter integrity and its relationship with outcome. J Neurotrauma 2018;35:2365-76 CrossRef Medline

8. Chung S, Fieremans E, Wang X, et al. White matter tract integrity: an indicator of axonal pathology after mild traumatic brain injury. J Neurotrauma 2018;35:1015-20 CrossRef Medline

9. Marmarou A, Foda MA, van den Brink W, et al. A new model of diffuse brain injury in rats, Part I: pathophysiology and biomechanics. J Neurosurg 1994;80:291-300 Medline

10. Mountney A, Boutte AM, Cartagena CM, et al. Functional and molecular correlates after single and repeated rat closed-head concussion: indices of vulnerability after brain injury. $\mathrm{J} \mathrm{Neu}$ rotrauma 2017;34:2768-89 CrossRef Medline

11. Kao YC, Li W, Lai HY, et al. Dynamic perfusion and diffusion MRI of cortical spreading depolarization in photothrombotic ischemia. Neurobiol Dis 2014;71:131-39 CrossRef Medline

12. Kao YJ, Oyarzabal EA, Zhang $\mathrm{H}$, et al. Role of genetic variation in collateral circulation in the evolution of acute stroke: a multimodal magnetic resonance imaging study. Stroke 2017;48:754-61 CrossRef Medline

13. Lai HY, Albaugh DL, Kao YC, et al. Robust deep brain stimulation functional MRI procedures in rats and mice using an MR-compatible tungsten microwire electrode. Magn Reson Med 2015;73: 1246-51 CrossRef Medline

14. Kuo DP, Lu CF, Liou M, et al. Differentiation of the infarct core from ischemic penumbra within the first 4.5 hours, using diffusion tensor imaging-derived metrics: a rat model. Korean J Radiol 2017; 18:269-78 CrossRef Medline

15. Yarnell AM, Barry ES, Mountney A, et al. The Revised Neurobehavioral Severity Scale (NSS-R) for rodents. Curr Protoc Neurosci 2016; 75:9.52.1-9.52.16 CrossRef Medline

16. Hamm RJ. Neurobehavioral assessment of outcome following traumatic brain injury in rats: an evaluation of selected measures. $\mathrm{JNeu}$ rotrauma 2001;18:1207-16 CrossRef Medline

17. Robinson S, Winer JL, Berkner J, et al. Imaging and serum biomarkers reflecting the functional efficacy of extended erythropoietin treatment in rats following infantile traumatic brain injury. $\mathrm{J} \mathrm{Neu}$ rosurg Pediatr 2016;17:739-55 CrossRef Medline

18. Green EW, O'Callaghan EK, Hansen CN, et al. Drosophila circadian rhythms in seminatural environments: summer afternoon component is not an artifact and requires TrpA1 channels. Proc Natl Acad Sci U S A 2015;112:8702-07 CrossRef Medline

19. Acabchuk R, Briggs DI, Angoa-Perez M, et al. Repeated mild traumatic brain injury causes focal response in lateral septum and hippocampus. Concussion 2016;1 CrossRef Medline

20. Namjoshi DR, Good C, Cheng WH, et al. Towards clinical management of traumatic brain injury: a review of models and mechanisms from a biomechanical perspective. Dis Model Mech 2013;6:1325-38 CrossRef Medline

21. Shultz SR, McDonald SJ, Vonder Haar C, et al. The potential for animal models to provide insight into mild traumatic brain injury: 
translational challenges and strategies. Neurosci Biobehav Rev 2017; 76(Pt B):396-414 CrossRef Medline

22. Xiong Y, Mahmood A, Chopp M. Animal models of traumatic brain injury. Nat Rev Neurosci 2013;14:128-42 CrossRef Medline

23. Goddeyne $\mathrm{C}$, Nichols J, Wu C, et al. Repetitive mild traumatic brain injury induces ventriculomegaly and cortical thinning in juvenile rats. J Neurophysiol 2015;113:3268-80 CrossRef Medline

24. Shultz SR, Bao F, Omana V, et al. Repeated mild lateral fluid percussion brain injury in the rat causes cumulative long-term behavioral impairments, neuroinflammation, and cortical loss in an animal model of repeated concussion. J Neurotrauma 2012;29:281-94 CrossRef Medline

25. Meconi A, Wortman RC, Wright DK, et al. Repeated mild traumatic brain injury can cause acute neurologic impairment without overt structural damage in juvenile rats. PLoS One 2018;13:e0197187 CrossRef Medline

26. McKee AC, Stein TD, Kiernan PT, et al. The neuropathology of chronic traumatic encephalopathy. Brain Pathol 2015;25:350-64 CrossRef Medline

27. Tambalo S, Peruzzotti-Jametti L, Rigolio R, et al. Functional magnetic resonance imaging of rats with experimental autoimmune encephalomyelitis reveals brain cortex remodeling. J Neurosci 2015; 35:10088-100 CrossRef Medline

28. Borja MJ, Chung S, Lui YW. Diffusion MR imaging in mild traumatic brain injury. Neuroimaging Clin N Am 2018;28:117-26 CrossRef Medline

29. Budde MD, Janes L, Gold E, et al. The contribution of gliosis to diffusion tensor anisotropy and tractography following traumatic brain injury: validation in the rat using Fourier analysis of stained tissue sections. Brain 2011;134:2248-60 CrossRef Medline

30. Long JA, Watts LT, Chemello J, et al. Multiparametric and longitudinal MRI characterization of mild traumatic brain injury in rats. J Neurotrauma 2015;32:598-607 CrossRef Medline

31. Tu TW, Williams RA, Lescher JD, et al. Radiological-pathological correlation of diffusion tensor and magnetization transfer imaging in a closed head traumatic brain injury model. Ann Neurol 2016;79: 907-20 CrossRef Medline

32. Wang ML, Yu MM, Yang DX, et al. Longitudinal microstructural changes in traumatic brain injury in rats: a diffusional kurtosis imaging, histology, and behavior study. AJNR Am J Neuroradiol 2018; 39:1650-56 CrossRef Medline

33. Wright DK, Trezise J, Kamnaksh A, et al. Behavioral, blood, and magnetic resonance imaging biomarkers of experimental mild traumatic brain injury. Sci Rep 2016;6:28713 CrossRef Medline

34. Salo RA, Miettinen T, Laitinen T, et al. Diffusion tensor MRI shows progressive changes in the hippocampus and dentate gyrus after status epilepticus in rat: histological validation with Fourier-based analysis. Neuroimage 2017;152:221-36 CrossRef Medline

35. Sizonenko SV, Camm EJ, Garbow JR, et al. Developmental changes and injury induced disruption of the radial organization of the cortex in the immature rat brain revealed by in vivo diffusion tensor MRI. Cereb Cortex 2007;17:2609-17 CrossRef Medline

36. Williams G, Morris ME, Schache A, et al. Incidence of gait abnormalities after traumatic brain injury. Arch Phys Med Rehabil 2009;90: 587-93 CrossRef Medline

37. Papa L, Brophy GM, Welch RD, et al. Time course and diagnostic accuracy of glial and neuronal blood biomarkers GFAP and UCH-L1 in a large cohort of trauma patients with and without mild traumatic brain injury. JAMA Neurol 2016;73:551-60 CrossRef Medline

38. Kulbe JR, Geddes JW. Current status of fluid biomarkers in mild traumatic brain injury. Exp Neurol 2016;275(Pt 3):334-52 CrossRef Medline

39. Shahim P, Tegner Y, Gustafsson B, et al. Neurochemical aftermath of repetitive mild traumatic brain injury. JAMA Neurol 2016;73: 1308-15 CrossRef Medline

40. Yu F, Shukla DK, Armstrong RC, et al. Repetitive model of mild traumatic brain injury produces cortical abnormalities detectable by magnetic resonance diffusion imaging, histopathology, and behavior. J Neurotrauma 2017;34:1364-81 CrossRef Medline 